Ozcelik G., Becerik-Gerber B., Ghahramani A., and Wang Y. (2017). "Can Immersive Virtual Environments Be Used for Understanding Occupant-System Interactions Under Thermal Stimuli?” In: LC3 2017: Volume I - Proceedings of the Joint Conference on Computing in Construction (JC3), July 4-7, 2017, Heraklion, Greece, pp. 357-364. DOI: https://doi.org/10.24928/JC3-2017/0022.

\title{
CAN IMMERSIVE VIRTUAL ENVIRONMENTS BE USED FOR UNDERSTANDING OCCUPANT-SYSTEM INTERACTIONS UNDER THERMAL STIMULI?
}

\author{
Gokce Ozcelik $^{1}$, Burcin Becerik-Gerber ${ }^{2}$, Ali Ghahramani ${ }^{3}$, Yuchao Wang ${ }^{4}$
}

\begin{abstract}
Occupants' interactions with building systems, as well as occupant-related factors considerably influence a building's energy consumption. However, understanding occupant-system interactions related to thermal changes in built environments could be cumbersome due to the resources needed to create these environments or the resources needed for conducting controlled experiments in existing Physical Environments (PEs). One avenue is to use Immersive Virtual Environments (IVEs) where occupants' interactions with the built environment are measured in the context of thermal stimuli. However, for validating the adequacy of using IVEs for understanding occupant interactions with building systems and/or elements, it is imperative to first investigate if IVEs are proper representations of PEs. In this study, we benchmark IVEs to the PEs with regards to user perceptions relating to thermal stimuli. In a human subject experiment, we use surveys and subjective thermal votes both in the IVE and PE, where participants experience both hot and cold indoor thermal conditions. Perceived thermal comfort and satisfaction votes are analysed by using paired $t$-tests and ANOVA. The change parameters are defined for identifying the direction of perceived thermal comfort and satisfaction. Statistical inferences show that change in occupants' perceived thermal comfort and satisfaction in IVE and PE are not significantly different, and direction of the change is positive in majority of the cases (i.e., $100 \%$ of the participants were comfortable in PE, almost $95 \%$ of the participants were comfortable in IVE, $79 \%$ were satisfied in PE, $74 \%$ were satisfied in IVE at the end of the experiment).
\end{abstract}

Keywords: Immersive virtual environment; virtual reality; physical environment; thermal perception; building systems; occupant-building systems interactions

\section{INTRODUCTION}

In the United States, buildings account for approximately $42 \%$ of the total energy consumption, of which commercial buildings make up of almost half (DoE 2011). Heating, ventilation, and air conditioning (HVAC) systems are major contributors of this energy consumption with approximately $43 \%$ share, providing thermal comfort and air quality

1 PhD Student, Sonny Astani Dept. of Civil and Environmental Engineering, Viterbi School of Engineering, Univ. of Southern California, Los Angeles, CA. E-mail: gozcelik@usc.edu 2 Associate Professor, Sonny Astani Dept. of Civil and Environmental Engineering, Viterbi School of Engineering, Univ. of Southern California, Los Angeles, CA. E-mail: becerik@usc.edu, Tel: +1 213740 4383(Corresponding Author)

$3 \mathrm{PhD}$ Candidate, Sonny Astani Dept. of Civil and Environmental Engineering, Viterbi School of Engineering, Univ. of Southern California, Los Angeles, CA. E-mail: aghahram@usc.edu 4 Masters Student, Sonny Astani Dept. of Civil and Environmental Engineering, Viterbi School of Engineering, Univ. of Southern California, Los Angeles, CA. E-mail: yuchaowa@usc.edu 
(Pérez-Lombard, Ortiz \& Pout 2008). Not only building system operations but also occupants' interactions with building systems and occupant-related factors (i.e., occupant preferences, behaviour, habits) influence building energy consumption. Previous studies highlighted the gap between the simulated energy consumption predictions and actual measured building performances (Hong 2014). A key factor, limiting the ability of energy simulation models to predict building energy consumption, has been identified as user behaviour (Hong 2014), which is any response of an individual or groups to the environment. Obtaining micro-level information about occupant responses to thermal environments could lead to more accurate user energy consumption behaviour models for future user-centred building designs and better decision making with regards to energy consumption measures for operation of existing buildings.

Current thermal comfort standards (i.e., ANSI/ASHRAE Standard 55) are based on limited number of factors (e.g., climate, indoor air temperature, air humidity), and yet there exist numerous disregarded static and dynamic factors that are influential on personal thermal comfort (e.g., gender, age, acclimatization, food/beverage intake, past thermal experiences). While defining thermal comfort ranges for energy-centric environmental control strategies, it is worth mentioning that these standards started including the adaptive responses. Occupants' adaptive responses to a thermal environment could be physiological responses (e.g., changes in skin temperature due to acclimatization) and psychological responses (e.g., tolerating the thermal environment as is). Physical responses (i.e., occupant-system interactions), such as adjustment of a thermostat, are mediated by these physiological and psychological responses. Assessments on the nexus of external and internal determinants to understand the notion of perceived thermal comfort through occupant response are necessary. However, measuring these adaptive responses and understanding occupant-system interactions related to thermal changes in built environments could be cumbersome due to the resources needed to create these environments or the resources needed for conducting controlled experiments in existing PEs.

IVEs provide a multimodal sensory (i.e., visual, haptic, auditory, olfactory, thermal and gustatory, and any combination of them (Blascovich et al. 2002a)) experience to users, wherein they are perceptually enveloped (i.e., immersed) by continuous stream of stimuli and when users are enabled to interact with the environment (Witmer, Singer 1998). IVEs facilitate an increasing level of perceived realism without decreasing the experimental control, and also provide an enhanced control over the extraneous variables, while experimental studies in PEs potentially require resources (e.g., time and cost), and might have lower experimental control (Blascovich et al. 2002a). IVEs have been used in numerous studies in wide-range of fields (e.g., psychology, visual perception, education and training, psychotherapy, social psychology (Blascovich et al. 2002a)). Despite the wide use and acceptance of IVEs, and their ability of handling multimodal sensory cues, they have not been used for understanding occupant-system interactions and perceived thermal comfort in built environments. In fact, there are very few studies integrating the thermal stimuli to IVEs for enhancing the immersion experience (Hülsmann et al. 2014, Dionisio 1997a).

For validating the adequacy of using IVEs for understanding occupant-building systems and/or elements interactions, it is necessary to investigate whether IVEs are proper representations of PEs in the context of thermal comfort and perception. Therefore, in this study, we benchmark IVEs to PEs with regards to thermal stimuli mainly by subjective thermal vote measurements, and by exposing test subject to similar thermal stimuli in both an IVE and a PE. In this respect, understanding occupant behaviour in built 
environments for the development of unbuilt settings wherein experimenting contextual variations (e.g., changing layout of the built space, changing the view they are exposed to) with visual and corresponding thermal changes in the microclimate (e.g., providing thermal cues arising from these changes and occupants' interactions) would be possible. Extracting the missing occupant behaviour information through real-time experiential approaches would mediate devising futuristic data-driven building energy solutions, and provide a venue for future user-centred designs. Moreover, IVEs could provide cost- and resource- effective controlled experimental venues for understanding the triggers, frequency, sequence and patterns of human building interactions. Keeping in mind the widely-accepted definition of thermal comfort (i.e., the state of mind expressing the satisfaction with the thermal environment), it is worth mentioning that incorporating thermal perception of occupants would also help defining the position of body in this context, and help further understanding the body-mind interactions, and how IVEs impact these interactions.

\section{RESEARCH METHODOLOGY}

An experimental study was designed and conducted based on the fundamental design of experiments methods, through which hypothesis testing was enabled in a systematic procedure (i.e., a controlled experiment). Null hypothesis of the experiment is "Occupants' perceived thermal comfort and satisfaction are the same in the IVE and PE, when they receive thermal stimuli (i.e., experience hot and cold indoor temperature conditions) in the PE.” Thus, for understanding the adequacy of using IVEs in the thermal stimuli context, a randomized human subject experiment was designed and included physical environment sensing (i.e., ambient air temperature, and humidity), surveys (i.e., demographics, personality, presence, immersive tendencies), subjective thermal comfort and satisfaction votes. In order to compare perceived thermal comfort of occupants to thermal stimuli in IVEs and PEs, three parameters were measured: type of occupant-building system/element interaction (i.e., with a table fan, a radiant heater, a local heater and/or a cooler, a thermostat, beverages, clothes), perceived thermal comfort prior and subsequent to the interactions (e.g., participants were asked to report their thermal states on a 7 point Likert scale, satisfaction with the thermal environment prior and subsequent to the interactions on a 7 point Likert scale..

\subsection{Design of Experiments}

Experiments took place in an acclimatized multi-occupancy office space. Participants were exposed to hot or cold thermal stimuli in PEs while they were in the same office space in the IVE or PE. They were assigned a generic office task (i.e., a simple reading comprehension task) to be kept focused during the experiment. Occupant-building system interactions were observed, participants received surveys during and after the experiment. Subjective thermal comfort and satisfaction votes were collected per interaction. A multioccupancy office space (where participants were the only occupants) at the University of Southern California was used as the experimental setting. Participants were recruited in the presence of hot or cold thermal stimuli in PEs while they were in an office space in the IVE or PE (randomized order). The virtual office space was modelled and rendered in Revit 2015, 3ds Max, and Unity 3D, based on the scale dimensions of the physical office space. In order to ensure that the IVE is an adequate representative of the PE, IVE was modelled in a similar fashion (i.e., physical features of the model were the same in both IVE and PE) as PE (see Fig. 1). Participants were immersed in IVE through Oculus Rift Head Mounted 
Display. The workstation assigned to the participants was equidistant to the building systems and elements that they could interact with so as to eliminate the factor of 'ease of control' from their interactions. The workstation included a video display terminal, a keyboard, one local heater/cooler, one radiant heater, one table fan, and hot/cold beverages. The independent variable of the study was the indoor air temperature since occupants usually interact with thermostats to set indoor thermal conditions, and indoor air temperature is one of the most influential factor in thermal comfort. Season, indoor lighting intensity and configuration, context, interior design (including spatial definition of the occupant), ambient factors, activity levels were kept constant in all experimental treatments to isolate the thermal perceptions.

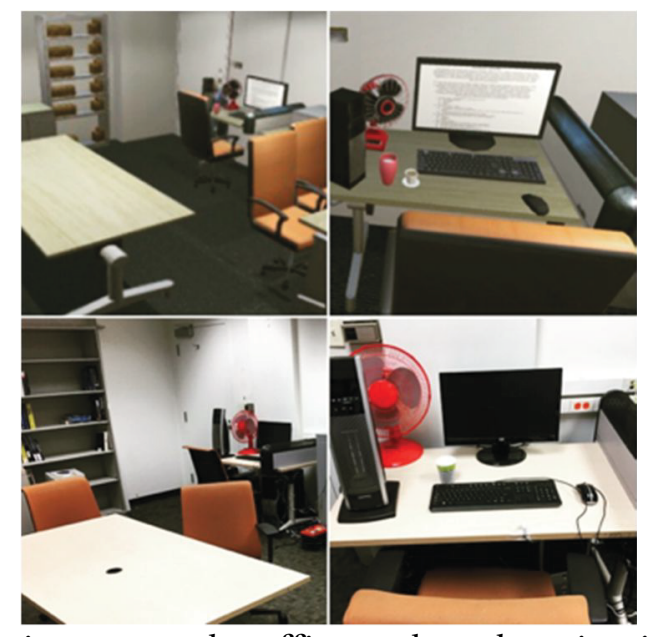

Fig. 1: Experiment environment: the office and workstation in IVE (top), the office and workstation in PE (bottom)

The results were obtained through the following systematic recruitment and procedure. 20 participants ( 7 females and 13 males) with random demographics were recruited in this pilot study. The participants were graduate and undergraduate students, who participated voluntarily or received extra credit through their program for their participation. Prior to the experiment, they received the Simulator Sickness questionnaire, went through an IVE training, and any participant with motion sickness was dismissed from the study. They were also informed that they can withdraw from the study at any time. Prior to starting the experiment, IRB-approved consent forms were signed by the participants. The participants were recruited in two groups; (1) hot indoor climate (i.e., 28 Celsius degrees), and (2) cold indoor climate (i.e., 18 Celsius degrees) in the PE and IVE. Due to the time limitations and hardship of changing thermal characteristics of the indoor environment, each day was designated for one of the two identified experiment groups. Groups were assigned to days randomly to eliminate the chance factor regarding the outside temperature of the day. The participants went through an information session on IVEs, during which they were trained for using the controller and interacting with the environment. The information session took place in another room with normal room temperature than the experiment setting. In any reported motion sickness case, participants were withdrawn from the study. In the same room, participants completed a demographics and personal information survey. Afterwards, they were welcomed to the experiment setting where they received the thermal stimuli.

Participants took Simulator Sickness questionnaire before (i.e., during the information session) and after the IVE sessions (i.e., at the same time as the subjective thermal votes). 
We also administered the presence questionnaires (i.e., Slater-Usoh-Steed, and Witmer and Singer presence questionnaires) to measure their presence in IVEs, and Immersive Tendencies questionnaire(ITQ) after the IVE session to measure the variations in tendencies of participants to experience presence, and the post-experiment survey (which included the perceived thermal comfort and satisfaction votes per interaction, and reasons of the interactions). Participants also took the Slater-Usoh-Steed presence questionnaire, and the post-experiment survey after the $\mathrm{PE}$ session of the experiment. These questionnaires were used for investigating the relationships among the reported presence and other research variables.

Participants were provided with equal number of interaction options per environment. These were adjusting the thermostat, adjusting the desk fan, adjusting the local heater, adjusting the local cooler, drinking hot beverages, drinking cold beverages, and adjusting the clothing level. In each session, the participants were requested to perform office activities (i.e., to read a passage and answer the questions related to the passage). They were informed that they could set the environment to their desired thermal comfort levels by interacting with building systems and/or elements in the environment. They were asked about their perceived thermal comfort levels, before, and just after their first-time interaction with one of the above-mentioned components. Participants were allowed to interact with the same component multiple times, but they received the survey for only the first-time interaction with that component. They reported their thermal comfort and satisfaction through subjective thermal votes at the end of each session (i.e., IVE and PE sessions) per first time-interactions with the components. At all times, indoor air temperature was recorded by SMAKN DHT22 AM2302 digital temperature and humidity measurement sensor.

\section{ANALYSIS AND DISCUSSION OF THE RESULTS}

One participant was not able to complete the experiment due to motion sickness. Thus, 19 participants' data (i.e., 10 participants in hot environment, 9 participants in cold environment) were used for the analysis. The results show that given the option to interact with their thermal environment, occupants made themselves comfortable both in PE and IVE, and there existed a significant improvement in their perceived thermal comfort. For analysing the perceived thermal comfort in each environment (i.e., PE hot condition, PE cold condition, IVE hot condition, IVE cold condition), participants' thermal comfort votes prior to their initial interactions (i.e., representative of the very first sensory impressions of occupants when they are exposed to a hot or cold microclimate before they had any interaction), and subsequent to their final interactions (i.e., representative of the participants' final thermal perceptions after they had interaction(s) to make themselves comfortable) were compared. Between initial and final interactions some participants were observed to perform multiple interactions. $97 \%$ of the perceived thermal comfort votes were 'comfortable' subsequent to the final interaction (Table 1). One tailed paired t-tests were performed to statistically compare the participants' perceived thermal comfort prior to their initial interactions and subsequent to their final interactions. We used $95 \%$ confidence level (i.e., alpha equals to 0.05 ) for the hypothesis test threshold. In other words, if $\mathrm{p}$-values are below 0.05 , there exists statistically significant difference between samples. The $\mathrm{p}$-values show that there exists significant difference between the means of perceived thermal comfort prior and subsequent to occupant-system interactions in all environments (Table 1). 
These results show that given the option to interact with the building systems/elements, participants were comfortable after their interactions both in the IVE and PE (please refer to the \% 'comfortable' vote subsequent to the last interaction column of Table 1 ). In addition to this, comfort votes of participants prior to their interactions were also analysed as representation of their very first sensory impressions (please refer to the \% 'comfortable' vote prior to the first interaction column of Table 1). In all environments, percentage of 'comfortable' votes increased, indicating the improvement of perceived thermal comfort of the participants. Further analysis showed that satisfaction with thermal environment prior and subsequent to occupant-system interactions are also significantly different for all environments but cold IVE (Table 1). The perceived satisfaction votes (i.e., percentage of participants who were 'satisfied' as tabulated in Table 1) also support these results. It is observed that in cold IVEs prior to the first interactions, $66.67 \%$ of the participants were satisfied whereas subsequent to the last interactions $55.56 \%$ which is why a significant difference in satisfaction was not observed in this environment, and a larger sample size is required for further inferences.

Table 1: Summary of the perceived thermal comfort and satisfaction results

\begin{tabular}{ccccccc}
\hline $\begin{array}{c}\text { Thermal } \\
\text { condition }\end{array}$ & $\begin{array}{c}\text { p-value } \\
\text { (perceived } \\
\text { thermal } \\
\text { comfort) }\end{array}$ & $\begin{array}{c}\text { p-value } \\
\text { (perceived } \\
\text { thermal } \\
\text { satisfaction) }\end{array}$ & $\begin{array}{l}\text { Comfortable } \\
\text { votes prior to } \\
\text { first } \\
\text { interaction }\end{array}$ & $\begin{array}{l}\text { Comfortable } \\
\text { votes } \\
\text { subsequent to } \\
\text { last } \\
\text { interaction }\end{array}$ & $\begin{array}{c}\text { Satisfied } \\
\text { votes prior } \\
\text { to first } \\
\text { interaction }\end{array}$ & $\begin{array}{c}\text { Satisfied } \\
\text { votes } \\
\text { subsequent } \\
\text { to last } \\
\text { interaction }\end{array}$ \\
\hline Hot PE & $0.0001^{*}$ & $0.0006^{*}$ & $20 \%$ & $100 \%$ & $10 \%$ & $80 \%$ \\
Cold PE & $0.0067^{*}$ & $0.0020^{*}$ & $44.44 \%$ & $100 \%$ & $11.11 \%$ & $77.78 \%$ \\
Hot IVE & $0.0406^{*}$ & $0.0007^{*}$ & $60 \%$ & $90 \%$ & $20 \%$ & $90 \%$ \\
Cold IVE & $0.0004^{*}$ & 0.2971 & $22.22 \%$ & $100 \%$ & $66.67 \%$ & $55.56 \%$ \\
\hline
\end{tabular}

*indicates the significant values

In order to compare the perceived thermal comfort in PEs to IVEs for understanding the adequacy of using IVEs in thermal comfort research, a change in thermal comfort parameter $(\Delta$ comfort) is defined and computed for PE (i.e., ( $\Delta$ comfort, PE) and IVE (i.e., $\Delta$ comfort, IVE). Qualitative thermal comfort and satisfaction votes were converted into quantitative data by enumerating and modifying the 7-Likert scale for statistically comparing the change in the direction of comfort and satisfaction. The third step was to enumerate the votes. In order to identify the direction change of comfort and satisfaction, negative votes (i.e., uncomfortable, dissatisfied) were enumerated as ' -1 ', positive votes (i.e., comfortable, satisfied) were enumerated as ' +1 '. These values were used to compute the $\Delta$ (i.e., change) parameters. This step-wise vote reduction methodology was only followed for identifying the direction of the change of comfort and satisfaction, for any other granular analysis 7-Likert scale was used as is. $\Delta$ comfort terms computed for the differences between the final (i.e., subsequent to the interactions) and initial (i.e., prior to the interactions) perceived thermal comfort votes in the PE and IVE separately. Afterwards, one-way analysis of variance (i.e., ANOVA) was performed on the two samples (i.e., PE and IVE) to test the null hypothesis (i.e., there exist no statistically significant difference between the perceived thermal comfort in PEs, and IVEs). Statistical analysis showed that $\mathrm{p}$-value is $0.3327>0.05$, and as hypothesized there exist no significant difference between 
perceived thermal comfort in PEs and IVEs. Thus, it was concluded that $(\Delta$ comfort, PE) $\approx$ $(\Delta$ comfort, IVE) in the context of thermal stimuli. ( $\Delta$ satisfaction) parameters were also computed in a similar fashion by pursuing the same methodology and p-value was found to be $0.0590>0.05$. However, increased sample size is needed to be able to arrive into any concrete conclusions.

As part of the post-experiment survey, participants were requested to report what they thought the ambient room temperature was before and after their interactions. They were provided 7 ranges of 5 Fahrenheit degrees each (e.g., 55-60 F, 60-65 F, and so on). The data for each participant's reported sense of initial room temperature in the PE and IVE (i.e., prior to their initial interactions) were compared. Although the same participant was welcomed to the same temperature range in both the PE and IVE, almost $16 \%$ of the participants reported lower temperatures in the PE than IVE, while $26 \%$ of the participants reported lower temperatures in the IVE than PE. A conclusion that could be derived from these results is that participants tend to feel cooler in IVEs than PEs. These findings need to be further pursued with a larger sample size for accurate inferences. In addition to these, ITQ and presence questionnaires were also analysed, descriptive statistics were obtained. Presence in PE was compared to IVE by one-way ANOVA ( $p=0.0097<0.05)$, and the reported presence in PE is significantly different than that of IVE. Furthermore, type of first interactions was also analysed and results were summarized in Table 2.

In the hot PEs and IVEs, the participants were observed to interact with the desk fan and the local heater first, while in the cold PEs and IVEs, they tended to interact with the local cooler and the thermostat first. First interactions are crucial since they represent the initial physical response and initial decision point of the occupants when they perceive a thermal stimulus in the environment. For further statistical analysis of demographics, personality, presence, and interaction type data, there is a need for a larger sample size.

Table 2: Summary of participants' first interactions

\begin{tabular}{cc}
\hline $\begin{array}{c}\text { Thermal } \\
\text { condition }\end{array}$ & First interactions (\% of participants) \\
\hline Hot PE & Local heater off (60\%), desk fan on (20\%), adjusting (decreasing) clothing level (10\%), \\
no interaction (10\%)
\end{tabular}

\section{CONCLUSION}

In this pilot study, occupants' perceptions about thermal comfort and satisfaction were measured in PEs and hybridized PE-IVEs for both hot and cold conditions to test the adequacy of using IVEs in thermal comfort studies. In order to better understand the direction of perceived thermal comfort and satisfaction, comfort and satisfaction change parameters were defined prior to initial occupant-building system/element interaction and subsequent to the final interactions. A mean comparison was performed by one-way ANOVA on change parameters. Analysis of $\left(\Delta_{\text {comfort }}\right)$ and $\left(\Delta^{\text {satisfaction }}\right)$ showed that, as hypothesized, there exist no significant difference between perceived thermal comfort and 
satisfaction in PEs and IVEs. IVEs could potentially be used in the context of thermal stimuli, however, due to the small sample size, we cannot make any conclusions.

The primary limitation is the small sample size; 20 participants were recruited in this study and 19 participants' data were analysed. For more in depth statistical inferences, sample size needs to be increased (to be determined by $99 \%$ power, 0.5875 effect size, $95 \%$ confidence). Our ongoing efforts include in designing a larger scale experiment with larger sample size, and enriched data collection to better understand the adequacy of using IVEs in the context of thermal stimuli. The novel observation of occupants' tendency of feeling cooler in IVEs could also be better understood in a larger sample. Future studies will also focus on understanding occupants' response to built environments where thermal and visual stimuli co-exist (i.e., multi modal sensory input do exist). Although the sample size does not allow us to do more in depth statistical analysis to further understand the similarities and differences between IVEs and PEs, the results of this pilot study are promising for focusing on validating the adequacy of using IVEs for understanding occupant- building system/element interactions in the context of thermal stimuli.

\section{ACKNOWLEDGEMENTS}

This material is based upon work supported by the National Science Foundation under Grant No. 1351701. Any opinions, findings, and conclusions or recommendations expressed in this material are those of the authors and do not necessarily reflect the views of the National Science Foundation.

\section{REFERENCES}

Blascovich, J., Loomis, J., Beall, A.C., Swinth, K.R., Hoyt, C.L. \& Bailenson, J.N. 2002a, "Immersive virtual environment technology as a methodological tool for social psychology", Psychological Inquiry, vol. 13, no. 2, pp. 103-124.

Dionisio, J. 1997a, "Temperature feedback in virtual environments", Advanced Imaging and Network TechnologiesInternational Society for Optics and Photonics, , pp. 233.

DoE, U. 2011, "Buildings energy databook", Energy Efficiency \& Renewable Energy Department, .

Hong, T. 2014, "Occupant behavior: impact on energy use of private offices", ASim 20121st Asia conference of International Building Performance Simulation Association., Shanghai, China, 11/25/12-11/27/12.

Hülsmann, F., Fröhlich, J., Mattar, N. \& Wachsmuth, I. 2014, "Wind and warmth in virtual reality: implementation and evaluation", Proceedings of the 2014 Virtual Reality International ConferenceACM, , pp. 24.

Pérez-Lombard, L., Ortiz, J. \& Pout, C. 2008, "A review on buildings energy consumption information", Energy and Buildings, vol. 40, no. 3, pp. 394-398.

Slater, M. \& Usoh, M. 1993, "Representations systems, perceptual position, and presence in immersive virtual environments", Presence: Teleoperators \& Virtual Environments, vol. 2, no. 3, pp. 221-233. 